\title{
Surgery Management of Pancreatitis with Complication: A Review Article
} \author{
Hernalia Martadila Putri ${ }^{4}$, \\ ${ }^{1}$ Department of Health, Faculty of Vocational Studies, Universitas Airlangga, Surabaya, Indonesia. \\ ${ }^{2}$ Faculty of Dental Medicine, Universitas Airlangga, Surabaya, Indonesia. \\ ${ }^{3}$ Department of Dental Nursing, Poltekkes Kemenkes, Surabaya, Indonesia. \\ ${ }^{4}$ Faculty of Medicine, Universitas Airlangga, Surabaya, Indonesia.
}

Nanda Rachmad Putra Gofur ${ }^{1 *}$, Aisyah Rachmadani Putri Gofur ${ }^{2}$, Soesilaningtyas ${ }^{3}$, Rizki Nur Rachman Putra Gofur ${ }^{4}$, Mega Kahdina ${ }^{4}$,

*Corresponding Author: Nanda Rachmad Putra Gofur, Department of Health, Faculty of Vocational Studies, Universitas Airlangga, Surabaya, Indonesia.

\section{Received Date: December 092021 | Accepted Date: 28 December 2021 | Published Date: 06 January 2022}

Citation: N R P Gofur, A R P Gofur, Soesilaningtyas, R N R P Gofur, M Kahdina. et al. (2022). Surgery Management of Pancreatitis with Complication: A Review Article. Endocrinology and Disorders. 6(1): DOI:10.31579/2640-1045/111

Copyright: () 2022 Nanda Rachmad Putra Gofur, This is an open-access article distributed under the terms of the Creative Commons Attribution License, which permits unrestricted use, distribution, and reproduction in any medium, provided the original author and source are credited.

\begin{abstract}
Introduction: Acute pancreatitis is an inflammatory disease of the pancreas with clinical manifestations that vary from mild to severe manifestations to death. The incidence of pancreatitis varies in various countries in the world and depends on the cause such as alcohol, gallstones, and metabolic factors. The clinical picture and the main symptom in patients with acute pancreatitis is abdominal pain. Abdominal pain varies from mild to severe and excruciating. Abdominal pain that is felt is constant and dull, and is usually felt in the epigastrium and periumbilicus and often spreads to the back, chest, waist, and lower abdomen.

Discussion: The onset of acute pancreatitis, the patient should be evaluated for hemodynamic status immediately and receive the necessary resuscitation measures. Patients with acute pancreatitis should receive aggressive intravenous rehydration $(250-500 \mathrm{ml} / \mathrm{hour}$ with isotonic crystalloid fluid) as early as possible with close monitoring, unless contraindicated with cardiovascular and/or renal comorbidities. It is most effective within the first 12-24 hours, but after that the benefits may diminish Debridement (necrosectomy) is the gold standard in infected acute necrotizing pancreatitis and peripancreatic necrosis. Indications for intervention either through radiological, endoscopic or surgical procedures in necrotizing pancreatitis are suspected or proven infected necrotizing pancreatitis with clinical deterioration, especially after the necrotic tissue has been encapsulated with thick walls (walled-off necrosis). Sterile necrotizing pancreatitis with persistent organ failure several weeks after the onset of acute pancreatitis, particularly after the necrotic tissue has been encapsulated with thick walls (walledoff necrosis).

Conclusion: Surgical management is often used in pancreatitis associated with gallstones. Cholecystectomy within 48 hours of the complaint can increase healing time. In addition, cholecystectomy performed early may not increase the risk of complications secondary to surgery. Surgery is not performed in acute necrotizing pancreatitis until the inflammation is reduced and the fluid accumulation no longer increases in size.
\end{abstract}

Keywords: complication; pancreatitis; surgery; management

\section{Introduction}

Acute pancreatitis is an inflammatory disease of the pancreas with clinical manifestations that vary from mild to severe manifestations to death. The incidence of pancreatitis varies in various countries in the world and depends on the cause such as alcohol, gallstones, and metabolic factors.
In the UK the incidence of acute pancreatitis reaches 5.4/100,000 per year, in America the incidence is higher at about 79.8/100,000 per year so that there are about 210,000 new cases per year, $20 \%$ of these cases are severe acute pancreatitis that is necrotizing. Disease and the overall mortality rate is around $10 \%$. This prevalence has increased in recent years [1]. 
The clinical picture and the main symptom in patients with acute pancreatitis is abdominal pain. Abdominal pain varies from mild to severe and excruciating. Abdominal pain that is felt is constant and dull, and is usually felt in the epigastrium and periumbilicus and often spreads to the back, chest, waist, and lower abdomen. The pain will increase if you are in a supine position and when you are given food or fluid intake. And the patient will feel more comfortable if sitting bent and knees bent. Other frequent patient complaints are nausea, vomiting, abdominal distension due to gastric and intestinal hypomotility and chemical peritonitis [2].

Patients with severe acute pancreatitis should be given enteral nutrition immediately to prevent infectious complications (avoiding prolonged fasting). Meanwhile, parenteral nutrition can continue if there is no access to enteral nutrition, cannot tolerate enteral nutrition or the caloric needs with enteral nutrition are inadequate.Stable patients with infected necrosis should delay surgical intervention, radiological and/or endoscopic drainage, preferably for 4 weeks, this allows time for the surrounding wall of necrosis to develop. Surgical management is often used in pancreatitis associated with gallstones [2, 3].

\section{Discussion}

The occurrence of acute pancreatitis is based on the presence of autodigestion of organs resulting from the activation of pancreatic enzymes. Pancreatitis is triggered by various mechanisms, including pancreatic duct obstruction, pancreatic ischemia, toxins, immunology and can be influenced by metabolic factors. There are 3 major pathological processes in the acinar cells that initiate injury to the pancreas. First, zymogen activation and early activation of other pancreatic digestive enzymes. Second, activation, chemoattraction and sequestration of neutrophils in the pancreas that cause intra-pancreatic inflammatory reactions of varying severity, and thirdly, the pancreas will produce pro-inflammatory mediators $[2,4]$.

The most important protective mechanism is the separation of zymogen granules by activated hydrolase. Theories that support this protective mechanism are the co-localization theory and the trypsinogen autoactivation theory. In acute pancreatitis, there is no separation so that there will be co-localization between zymogen and hydroloase which will activate intra acinar enzymes. Trypsinogen has the ability to activate and inhibit itself, if in a state of eating obstruction there will be an increase in the sensitivity of trypsinogen activation, and if this situation is not controlled, gland auto-digestion can occur [5].

Physical examination often found the patient looked restless and anxious. In addition, tachycardia, low-grade fever, and hypotension are common, leading to signs of shock. Jaundice is rare, but if there is jaundice it can be caused by edema of the head of the pancreas with compression of the common bile duct in the intrapancreas. Erythematous nodes may form on the skin as a result of subcutaneous fat necrosis $[2,6]$.

Approximately $10-20 \%$ of patients with acute pancreatitis can find pleural effusion, basal crackles, atelectasis and other pulmonary abnormalities, especially on the left side. Cullen's sign appears (Cullen's sign), which is discoloration around the umbilicus due to hemoperitoneum and Blue-redpurple Turner's sign in the waist due to catabolism of hemoglobin in the tissues. Both of these signs are rare, but if present they indicate the presence of severe necrotizing pancreatitis.

Plain abdominal radiographs are performed especially if there are indications of other abdominal diseases, but it can also be used to view images with the impression of acute pancreatitis. So it is possible to exclude the presence of other abdominal diseases, such as gastrointestinal perforation. Ultrasound of the abdomen is an important visualization in patients with abdominal pain associated with jaundice and for the exclusion of gallstones as a cause of acute pancreatitis. So ultrasound is useful to determine the etiology of acute pancreatitis. Abdominal ultrasound has a sensitivity of about $95 \%$ for detecting cholelithiasis and for choledocholithiasis only about $50 \%$ [2,3].

$\mathrm{CT}$ abdomen is useful for determining the severity and the presence or absence of complications from pancreatitis using the Balthazar score. In an inflamed pancreas, pancreatomegaly appears with flat edges, parenchyma is heterogeneous and pancreatic lines appear (peripancreatic steakiness) or dirty fat which is a visualization of peripancreatic fluid. Dynamic CT showed necrotic pancreatic parenchyma after intravenous contrast administration [7].

\section{Management of Pancreatitis}

The onset of acute pancreatitis, the patient should be evaluated for hemodynamic status immediately and receive the necessary resuscitation measures. Patients with acute pancreatitis should receive aggressive intravenous rehydration ( $250-500 \mathrm{ml} /$ hour with isotonic crystalloid fluid) as early as possible with close monitoring, unless contraindicated with cardiovascular and/or renal comorbidities. It is most effective within the first 12-24 hours, but after that the benefits may diminish [8].

Debridement (necrosectomy) is the gold standard in infected acute necrotizing pancreatitis and peripancreatic necrosis. Indications for intervention either through radiological, endoscopic or surgical procedures in necrotizing pancreatitis are suspected or proven infected necrotizing pancreatitis with clinical deterioration, especially after the necrotic tissue has been encapsulated with thick walls (walled-off necrosis). Sterile necrotizing pancreatitis with persistent organ failure several weeks after the onset of acute pancreatitis, particularly after the necrotic tissue has been encapsulated with thick walls (walled-off necrosis) [9].

Sterile acute necrotizing pancreatitis does not require surgery, it is quite conservative unless fulminant acute pancreatitis occurs. Indications for intervention in sterile necrotizing pancreatitis are obstruction of the biliary, intestinal or gastric outlet due to pressure of necrotic tissue and encapsulated fluid (walled-off necrosis), patients with walled-off necrosis without signs of infection but still experiencing persistent symptoms (eg abdominal pain), leakage syndrome pancreatic duct (disconnected duct) with persistent symptoms (eg pain or obstruction) with necrosis in the absence of infection (approximately $>8$ weeks after onset of acute pancreatitis) ${ }^{10}$.

Complications of acute pancreatitis are divided into organ failure and systemic complications and local complications. The organ systems assessed in relation to organ failure were respiration, heart and kidneys. The frequency of occurrence of organ failure in patients with severe acute pancreatitis was multiple organ failure $(27 \%)$, respiratory failure $(46 \%)$, renal failure $(16.2 \%)$, heart failure $(17.6 \%)$, liver failure $(18.9 \%)$, and gastrointestinal bleeding $(10.8 \%)$. The mortality rate from multiple organ failure is $45 \%$. Organ failure was defined as a score of 2 for one of the three organ systems using the Marshall scoring system. Systemic complications were assessed based on the presence of an exacerbation of pre-existing comorbidities, such as: coronary heart disease or chronic obstructive pulmonary disease, precipitated by acute pancreatitis $[2,5]$.

From a previous study of infected necrotizing pancreatitis treated operatively, delaying surgery reduced mortality by $22 \%$. Although patients with unstable necrotizing pancreatitis require immediate debridement, current consensus recommends that stable patients should be given antibiotics prior to intervention to suppress the inflammatory reaction. If the patient's condition has not improved and the infectious necrosis has not subsided, minimally invasive necrosectomy via radiology, endoscopy or laparoscopy should be considered [10].

A study of the open necrosectomy method versus the step-up approach (intervention of percutaneous drainage and, if necessary, minimally 
invasive retroperitoneal necrosectomy) in patients with infected necrotizing pancreatitis. They concluded that minimally invasive procedures in infected necrotizing pancreatitis reduced the major complications (organ failure, perforation). visceral organs or bleeding) and mortality compared to open surgery [1].

Mild acute pancreatitis with gallstones in the gallbladder, cholecystectomy should be performed before the patient is discharged from the hospital to prevent recurrence of acute pancreatitis (strong recommendation, moderate quality of evidence). In acute necrotizing biliary pancreatitis, to prevent infection, cholecystectomy is postponed until the active inflammation is gone and fluid accumulation improves or stabilizes (strong recommendation, moderate quality of evidence). The presence of asymptomatic pseudocysts and pancreatic and/or extra pancreatic necrosis does not require intervention, regardless of size, location, and/extension (strong recommendation, moderate quality of evidence) [11].

Stable infected necrosis, surgical, radiological, and/or endoscopic drainage should be delayed by more than 4 weeks, to allow liquefication of the contents and development of a fibrous wall around the necrosis (walled-off necrosis) (strong recommendation, low quality of evidence). In symptomatic infected necrosis, minimally invasive necrosectomy is preferred over open necrosectomy (strong recommendation, low quality of evidence). Surgical management through ERCP correlates with choledocholithiasis. However, the consensus suggests that ERCP is not routinely carried out right. In acute cholangitis or serum bilirubin $>5$ $\mathrm{mg} / \mathrm{dl}$ ERCP is still useful. ERCP can be used to identify pancreatic ductal disruption in severe acute pancreatitis and to intervene in ductal dislocation syndrome [12].

In general, interstitial edematous pancreatitis with fluid retention is resorbed within 7-10 days, only $6.8 \%$ of cases develop pseudocysts. Asymptomatic pseudocysts do not require intervention, but in their course they can change character to become symptomatic. If the pseudocyst is symptomatic, the treatment options are decompression via percutaneous drainage or endoscopic cyst gastrostomy with endoscopic ultrasound guidance. Open surgery is an option if the pseudocyst is complex, multiple, or there are complications such as fistula, rupture and bleeding [8].

\section{Conclusion}

Surgical management is often used in pancreatitis associated with gallstones. Cholecystectomy within 48 hours of the complaint can increase healing time. In addition, cholecystectomy performed early may not increase the risk of complications secondary to surgery. Surgery is not performed in acute necrotizing pancreatitis until the inflammation is reduced and the fluid accumulation no longer increases in size.

\section{References}

1. Kabbara Wissan K, Nawas George T, Ramadan Wijdan. (2015). Evaluation of the appropriateness of imipinem/cilastatin prescription and dosing in a tertiary care hospital. Infect Drug Resist. 8:31-39.

2. Zhu AJ, Shi JS, Sun XJ. (2003). Organ failure associated with severe acute pancreatitis. World J Gastroenterol. 9(11):25702573.

3. Xuong L, Elie Aoun. (2012). Complications of acute pancreatitis. Practical Gastroenterol. 11-21.

4. Bechien Wu, Peter AB. (2013). Clinical management of patients with acute pancreatitis. Gastroenterology. 144:1272-1281.

5. DiMagno MJ, DiMagno EP. (2007). New advances in acute pancreatitis. Curr Opin Gastroenterol. 23:494-501.

6. Akova M. (2008). Sulbactam-containing beta lactmase inhibitor combinations. Clin Microbiol Infect. 14(1):185-188.

7. Busireddy, Kirain K, Ramalho, miguel, Semelka, Richard S. (2014). Pancreatitis- imaging approach. World J of Gastrointest Pathophysiol. 15(3).

8. Chen-Qiju, Yong Yang, Zhi, You Wang, Chun, Ming Dong, Li. (2016). Hydroxyethyl starch resuscitation downregulate proinflammatory cytokines in the early phase of severe acute pancreatitis: A retrospective study. Exp Ther Med.12:3213-3220.

9. Kui, Parnizky, Azentesi A, Ballazs A, Scuzs A. (2016). Prosperctive, Mulitcentre Nationwide Clinical Data from 600 cases of Acute Pancreatitis. PLoS One. 11(10):1-19.

10. Topazian M, Pandol SJ. Acute Pancreatitis. In Yamada, Tadakata.,Alpers DH., kaloo An., Kaplowitz N, Owyang C, Powell DW. (2008). Textbook of gastroenterology. 1761-1810.

11. Greenberg, Joshua, Hsu, Jonathan, Bawazeer, Mohammad, Marshall, John, Friedrich, Jan O. (2014). Clinical practice guidelines: management of acute pancreatitis. J Can Chir.59(2):128-140.

12. Tenner S, Baillie J, Dewitt J, et al. (2013). American College of Gastroenterology Guidelines: Management of Acute Pancreatitis. Am J Gastroenterol. 108:1400-1415.

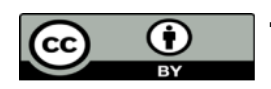

This work is licensed under Creative Commons Attribution 4.0 License
To Submit Your Article Click Here:

Submit Manuscript
Ready to submit your research? Choose Auctores and benefit from:

$>$ fast, convenient online submission

$>$ rigorous peer review by experienced research in your field

$>$ rapid publication on acceptance

$>$ authors retain copyrights

$>$ unique DOI for all articles

$>$ immediate, unrestricted online access

At Auctores, research is always in progress.

Learn more https://auctoresonline.org/journals/endocrinology-and-disorders
DOI: $10.31579 / 2640-1045 / 111$ 